\title{
Low prognostic nutrition index predicts poorer quality of life in late-stage lung cancer
}

\author{
Zhaohui Zhang $^{1 \#}$, Aiping Chen ${ }^{1 \#}$, Fenglan Xie ${ }^{2}$, Xuefei Li $^{1}$, Guoxiang Hu${ }^{1}$, Gengpeng Lin ${ }^{1}$ \\ ${ }^{1}$ Department of Pulmonary and Critical Care Medicine, The First Affiliated Hospital of Sun Yat-sen University, Institute of Pulmonary Diseases, Sun \\ Yat-sen University, Guangzhou, China; ${ }^{2}$ Department of Nursing, The First Affiliated Hospital of Sun Yat-sen University, Guangzhou, China \\ Contributions: (I) Conception and design: Z Zhang, G Lin; (II) Administrative support: A Chen, G Lin; (III) Provision of study materials or patients: F \\ Xie, X Li; (IV) Collection and assembly of data: X Li, G Hu; (V) Data analysis and interpretation: F Xie, G Lin; (VI) Manuscript writing: All authors; \\ (VII) Final approval of manuscript: All authors. \\ "These authors contributed equally to this work. \\ Correspondence to: Gengpeng Lin. Department of Pulmonary and Critical Care Medicine, The First Affiliated Hospital of Sun Yat-sen University, \\ Institute of Pulmonary Diseases, Sun Yat-sen University, No. 58 Zhongshan 2nd Road, Guangzhou, China. Email: lingp6@mail.sysu.edu.cn.
}

\begin{abstract}
Background: Quality of life (QOL) is one of the most important endpoints in lung cancer care. Both nutritional and immune status reportedly correlate with QOL, so we investigated whether the prognostic nutritional index (PNI), a reliable marker of nutritional and immune status, can predict QOL in late-stage lung cancer.

Methods: We enrolled 80 lung cancer patients and their clinical data including PNI were obtained. The FACT-L questionnaire in Chinese version 4 was administered to every patient.

Results: Of the 80 lung cancer patients, 16 were stage III and 64 were stage IV. The average PNI value was 44.24 \pm 5.53 . The average FACT-L score was $99.58 \pm 21.84$, indicating impaired QOL. The FACT-L score in the stage IV group was significantly lower than that in the stage III group $(\mathrm{P}=0.001)$, especially for the four subscales of physical, social/family, emotional, and functioning well-being. In the stage IV group, the FACT-L score in the high PNI group was significantly higher than that in the low PNI group $(\mathrm{P}=0.042)$, with especially higher score for the physical well-being subscale. PNI was significantly related to both the FACT-L score $(r=0.3265, \mathrm{P}=0.0085)$ and physical well-being subscale $(\mathrm{r}=0.4746, \mathrm{P}<0.0001)$.
\end{abstract}

Conclusions: PNI is a simple but valuable biomarker of QOL in stage IV lung cancer patients. A lower PNI may indicate the need for detailed QOL evaluation and intervention.

Keywords: Lung cancer; prognostic nutrition index (PNI); quality of life (QOL)

Submitted Aug 26, 2020. Accepted for publication Oct 27, 2020.

doi: 10.21037/apm-20-1892

View this article at: http://dx.doi.org/10.21037/apm-20-1892

\section{Introduction}

For decades, lung cancer has remained the most prevalent cancer worldwide with high morbidity and mortality rate $(1,2)$. In GLOBOCAN 2018, lung cancer was estimated to account for $11.6 \%$ of all new cancer cases, and $18.4 \%$ of the total cancer deaths (3). In China, the prevalence of lung cancer is still rising, but the mortality rate is decreasing because of advances in medical treatment and diagnosis in the past decade (4). Nevertheless, most lung cancer patients have a high burden of psychological suffering leading to poor quality of life (QOL), which is related to unfavorable outcomes $(5,6)$. Although it has been proved that high QOL is a positive predictor of prognosis in lung cancer $(7,8)$, the complex physical and psychological consequences of the disease that typically affect patients are still overlooked in the common disease-centered approach $(9,10)$. Consequently, QOL is an issue of importance for lung cancer patients, but its evaluation requires administration of tedious questionnaires such as EORTC QLQ-C30 and so 
is not routinely performed in daily clinical practice $(11,12)$. Thus, a simple but reliable biomarker of QOL in lung cancer patients would be helpful.

Nutritional status is related to QOL in lung cancer patients (13), and several parameters for evaluating nutritional status have been proposed, such as serum albumin, the prognostic nutritional index (PNI), body mass index (BMI) and the subjective global assessment (SGA) (14-16). The calculation of the PNI is based on the concentration of albumin and the lymphocyte count $[10 \times$ albumin $(\mathrm{g} / \mathrm{dL})+0.005 \times$ lymphocyte count $\left.\left(/ \mathrm{mm}^{3}\right)\right]$. Thus, the PNI comprehensively reflects both the nutritional and immune status of the patient and has proven reliable in predicting survival of lung cancer patients $(17,18)$. However, the predictive value of the PNI for the QOL of late-stage lung cancer patients is unclear, so we investigated the relationships among PNI, the clinicopathological characteristics and QOL in late-stage lung cancer patients.

We present the following article in accordance with the MDAR reporting checklist (available at http://dx.doi. org/10.21037/apm-20-1892).

\section{Methods}

\section{Study design and patients}

From January 2019 to December 2019, 80 patients with newly diagnosed lung cancer in the Department of Pulmonary and Critical Medicine of the First Affiliated Hospital of Sun Yat-sen University were included in the study. Inclusion criteria were as follows: diagnosis of histopathologically confirmed stage III or IV lung cancer; age 18-80 years; and Eastern Cooperative Oncology Group (ECOG) score $\leq 2$. Exclusion criteria included patients with poor functional performance status, who refused to take part in the study, who had received previous systemic treatment such as radiotherapy and/or chemotherapy, and patients with signs of acute infection. Clinical data were obtained after the patient's first admission to the Department, including medical history, blood cytology and chemistry, definitive histopathological diagnosis, and clinical tumornode-metastasis (TNM) stage (19), as well as ECOG score determined by the clinical oncologist.

All subjects gave written consent after they were fully informed of the study's objectives and methods. The study was approved by the Institutional Research Ethics Committee of the First Affiliated Hospital of Sun Yatsen University. All subjects were required to be able to independently complete a questionnaire. The study was conducted in accordance with the Declaration of Helsinki (as revised in 2013).

\section{QOL evaluation}

The QOL evaluation was performed at baseline, which was 7 days prior to initiation of systemic therapy. We used the Chinese version 4 of the FACT-L questionnaire, which has five domains (subscales): physical well-being (PWB, 7 items), social/family well-being (SFWB, 7 items), emotional well-being (EWB, 6 items), functioning well-being (FWB, 7 items) and additional concerns-lung (LCS, 9 items). The details of this questionnaire were described in a previous study (20).

\section{Statistical analysis}

All data analyses were performed using SPSS software (version 18.0) (SPSS Inc., Chicago, IL, USA). Continuous variables are shown as average means with standard deviation or median with range. Differences between groups were analyzed by the Student's $t$-test for those that were normally distributed, and by the Mann-Whitney $U$ test for those that were not. Multivariate analysis of factors associated with QOL used the linear multiple regression model. $\mathrm{P}<0.05$ was considered statistically significant. Statistical analyses were performed using GraphPad Prism5.

\section{Results}

\section{Characteristics of the study subjects}

The majority of the 80 late-stage lung cancer patients were male $(68.8 \%)$, with an average age of $61.20 \pm$ 11.01 years, mean BMI of $22.03 \pm 2.84 \mathrm{~kg} / \mathrm{m}^{2}$, mean serum albumin of $37.62 \pm 5.02 \mathrm{~g} / \mathrm{L}$, mean blood lymphocyte count of $(1.32 \pm 0.48) \times 10^{3} / \mathrm{mm}^{3}$ and mean hemoglobin of $118.34 \pm 21.56 \mathrm{~g} / \mathrm{L}$. The most common pathological type was adenocarcinoma $(66.3 \%)$. Most of the patients had an ECOG score 0-1 (84.09\%). The average PNI value was $44.24 \pm 5.53$, and the average FACT-L score was $99.58 \pm 21.84$, which indicated impaired QOL. Details are shown in Table 1.

\section{PNI and stage of lung cancer}

The patients were divided according to clinical stage into 
Table 1 Clinical characteristics of the lung cancer patients

\begin{tabular}{|c|c|}
\hline Parameter & $\mathrm{N}=80$ \\
\hline \multicolumn{2}{|l|}{ Gender } \\
\hline Male & 55 \\
\hline Female & 25 \\
\hline Age (years) & $61.20 \pm 11.01$ \\
\hline Height (m) & $1.65 \pm 0.08$ \\
\hline Weight (kg) & $60.06 \pm 9.85$ \\
\hline BMI $\left(\mathrm{kg} / \mathrm{m}^{2}\right)$ & $22.03 \pm 2.84$ \\
\hline \multicolumn{2}{|l|}{ Histology } \\
\hline Adenocarcinoma & 53 \\
\hline Squamous cell carcinoma & 19 \\
\hline Small-cell carcinoma & 6 \\
\hline Other & 2 \\
\hline \multicolumn{2}{|l|}{ TNM stage } \\
\hline III & 16 \\
\hline IV & 64 \\
\hline \multicolumn{2}{|l|}{ ECOG } \\
\hline 0 & 44 \\
\hline 1 & 30 \\
\hline$\geq 2$ & 6 \\
\hline Albumin (g/L) & $37.62 \pm 5.02$ \\
\hline Lymphocytes $\left(\times 10^{3} / \mathrm{mm}^{3}\right)$ & $1.32 \pm 0.48$ \\
\hline Hemoglobin (g/L) & $118.34 \pm 21.56$ \\
\hline PNI & $44.24 \pm 5.53$ \\
\hline FACT-L score & $99.58 \pm 21.84$ \\
\hline Physical well-being & $20.79 \pm 6.38$ \\
\hline Social/family well-being & $20.80 \pm 4.37$ \\
\hline Emotional well-being & $16.45 \pm 6.34$ \\
\hline Functioning well-being & $17.13 \pm 7.06$ \\
\hline Additional concerns-lung & $24.16 \pm 5.75$ \\
\hline
\end{tabular}

BMI, body mass index; ECOG, Eastern Cooperative Oncology

Group performance status; PNI, prognostic nutritional index.

stage III ( $n=16)$ and stage IV $(n=64)$. There were more male patients in the stage III group, but no significant differences were observed between the two groups with regard to age, sex, height, weight, and BMI. The pathological types and ECOG scores were similar between groups. There were

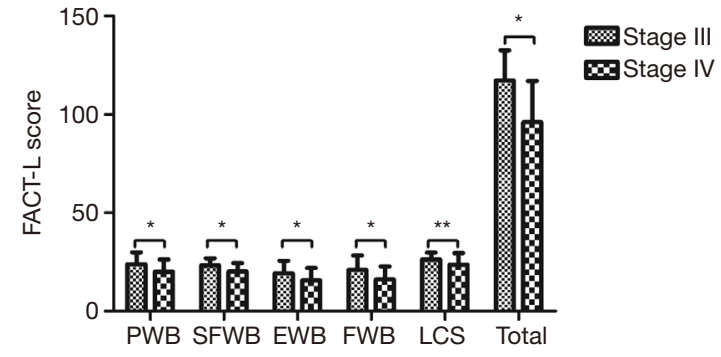

Figure 1 Comparison of FACT-L total score and each scale in stage III and stage IV lung cancer patients. * $\mathrm{P}<0.05 ;{ }^{* *}, \mathrm{P}>0.05$. PWB, physical well-being; SFWB, social/family well-being; EWB, emotional well-being; FWB, functioning well-being; LCS, additional concerns-lung; PNI, prognostic nutritional index.

no significant differences in serum album, hemoglobin, lymphocyte count or PNI. However, the FACT-L score in the stage IV group was significantly lower than that in the stage III group $(\mathrm{P}=0.001)$. The stage IV group had lower scores for the subscales of $\mathrm{PWB}, \mathrm{SFWB}, \mathrm{EWB}$, and FWB; however, no significant differences were found for the subscale of LCS (Figure 1, Table 2).

\section{PNI and QOL in stage IV lung cancer}

All stage IV lung cancer patients were divided into low PNI $(\leq 44, n=28)$ and high PNI $(>44, n=36)$. The high PNI group had higher levels of serum album, hemoglobin and lymphocytes (Figure 2). The FACT-L score in the high PNI group was significantly higher than that in the low PNI group ( $\mathrm{P}=0.042)$, with an especially higher score for the PWB subscale. However, no significant differences were observed for the other four subscales (Figure 3, Table 3).

\section{Correlation between PNI and FACT-L in stage IV lung cancer}

In the stage IV group of lung cancer patients, PNI was significantly related to FACT-L $(r=0.3265, \mathrm{P}=0.0085)$. Further correlation analysis showed that PNI was positively related to the subscale of $\mathrm{PWB}(\mathrm{r}=0.4746, \mathrm{P}<0.0001)$, but there was no statistical significance observed between PNI and SFWB, EWB, FWB or LCS (Figure 4).

\section{Determinants of $Q O L$}

Variables including age, sex, BMI, hemoglobin, and PNI were analyzed in a regression equation: $\mathrm{FACT}-\mathrm{L}$ score $=$ 
Table 2 Clinical characteristics of stage III and IV lung cancer patients

\begin{tabular}{|c|c|c|c|}
\hline Parameter & Stage III $(\mathrm{N}=16)$ & Stage IV (N=64) & $\mathrm{P}$ \\
\hline Male & 15 & 40 & \\
\hline Female & 1 & 24 & \\
\hline Age (years) & $65.06 \pm 9.92$ & $60.23 \pm 11.12$ & 0.117 \\
\hline Weight (kg) & $60.41 \pm 9.27$ & $59.98 \pm 10.06$ & 0.877 \\
\hline BMI $\left(\mathrm{kg} / \mathrm{m}^{2}\right)$ & $21.38 \pm 2.72$ & $22.20 \pm 2.87$ & 0.305 \\
\hline Histology & & & 0.013 \\
\hline Adenocarcinoma & 7 & 46 & \\
\hline Other & 2 & 0 & \\
\hline ECOG & & & 0.578 \\
\hline 0 & 9 & 35 & \\
\hline 1 & 7 & 23 & \\
\hline$\geq 2$ & 0 & 6 & \\
\hline Albumin (g/L) & $36.13 \pm 6.44$ & $37.99 \pm 4.59$ & 0.187 \\
\hline Lymphocytes $\left(\times 10^{3} / \mathrm{mm}^{3}\right)$ & $1.37 \pm 0.47$ & $1.31 \pm 0.48$ & 0.653 \\
\hline Hemoglobin (g/L) & $121.06 \pm 24.08$ & $117.66 \pm 21.04$ & 0.575 \\
\hline Functioning well-being & $21.06 \pm 7.29$ & $16.14 \pm 6.70$ & 0.012 \\
\hline Additional concerns-lung & $26.25 \pm 4.86$ & $23.64 \pm 5.87$ & 0.105 \\
\hline
\end{tabular}

BMI, body mass index; ECOG, Eastern Cooperative Oncology Group performance status; PNI, prognostic nutritional index.
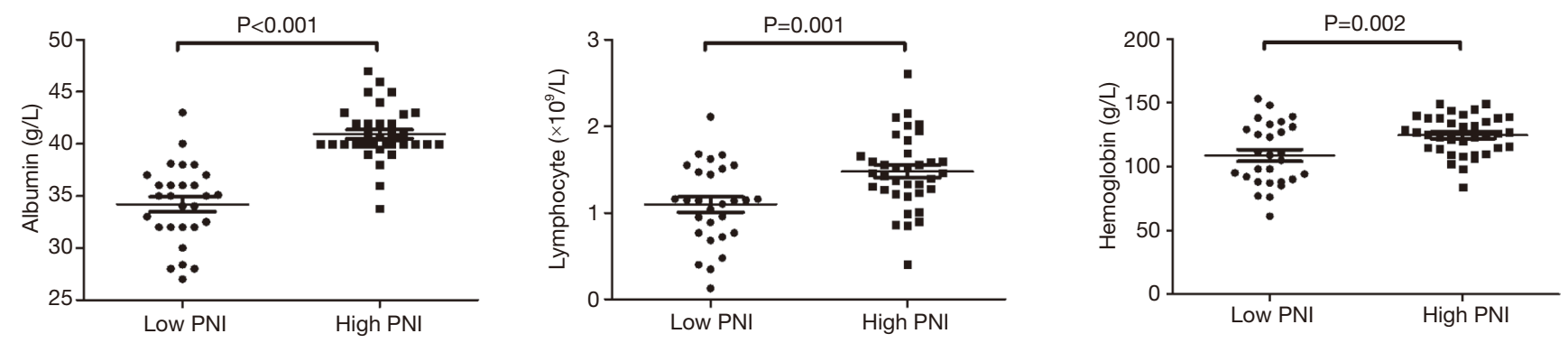

Figure 2 Comparison of nutritional parameters in lung cancer patients with low PNI and high PNI. PNI, prognostic nutritional index. 


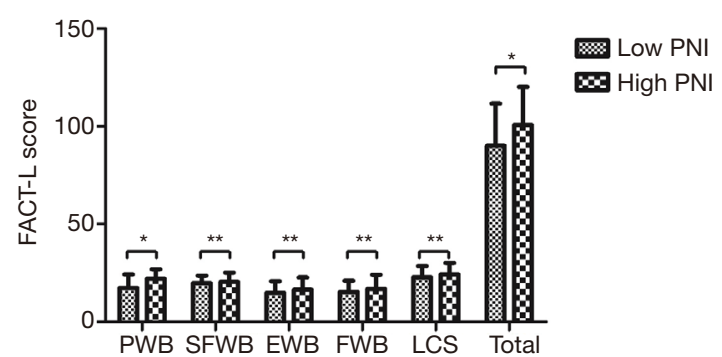

Figure 3 Comparison of FACT-L total score and each scale in lung cancer patients with low $\mathrm{PNI}$ and high $\mathrm{PNI}$. * $\mathrm{P}<0.05$; **, $\mathrm{P}>0.05$. PNI, prognostic nutritional index; $\mathrm{PWB}$, physical wellbeing; SFWB, social/family well-being; EWB, emotional wellbeing; FWB, functioning well-being; LCS, additional concernslung; PNI, prognostic nutritional index.
$37.128+1.322 \times$ PNI. PNI was an independent determinant of FACT-L score $(\mathrm{P}=0.008)$.

\section{Discussion}

In this study, we analyzed the clinical characteristics and QOL of late-stage lung cancer patients. In particular, we evaluated the relationship of PNI and QOL. The stage IV lung cancer patients had a lower FACT-L score than the stage III patients, and their PNI correlated with their FACT-L scores. Therefore, a low PNI could be a predictor of poorer QOL in this group of lung cancer patients.

QOL is defined as an individual's self-reported sense of well-being, and is measured by several scales such

Table 3 Clinical characteristics of stage IV lung cancer patients with low and high PNI

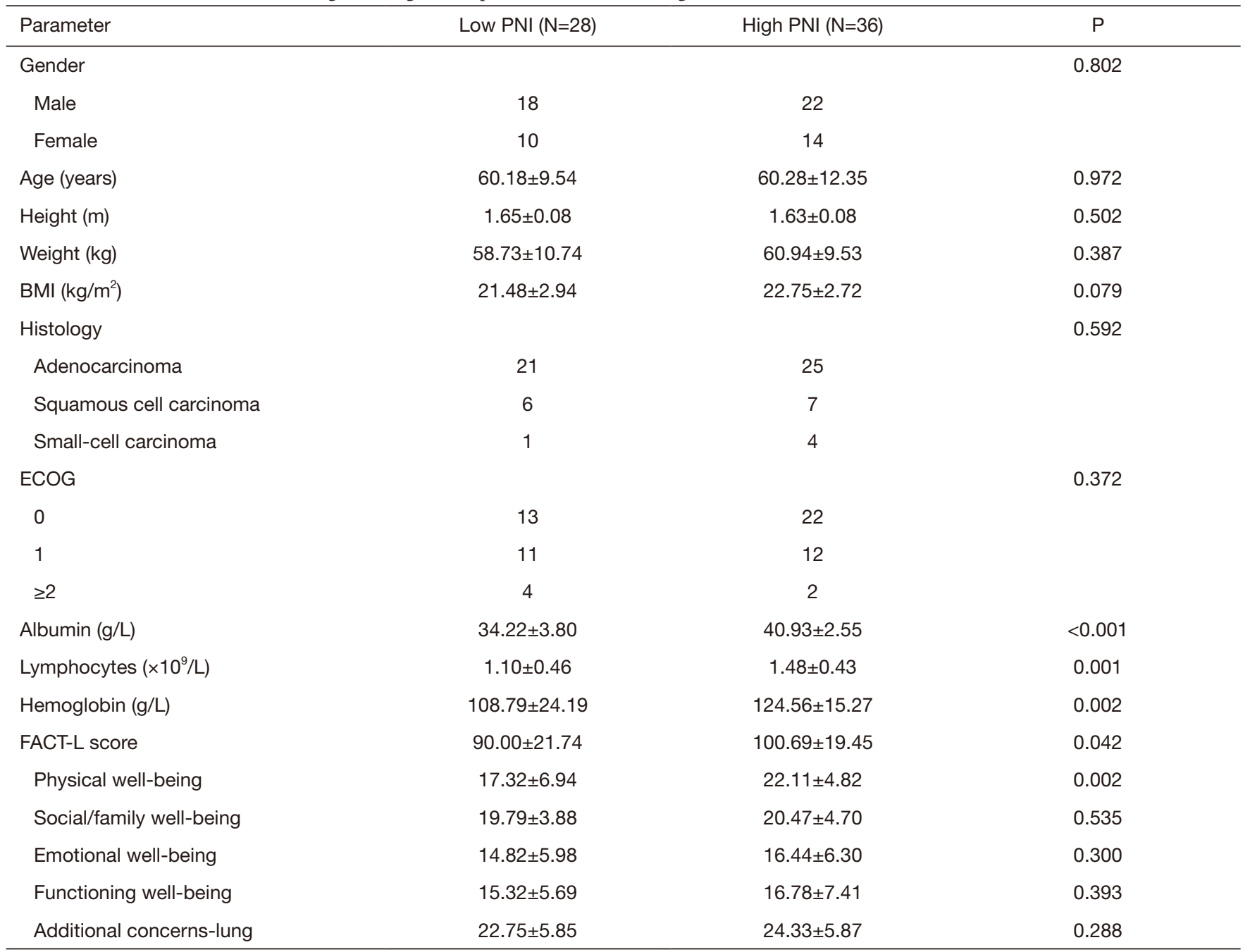

PNI, prognostic nutritional index; BMI, body mass index; ECOG, Eastern Cooperative Oncology Group performance status. 

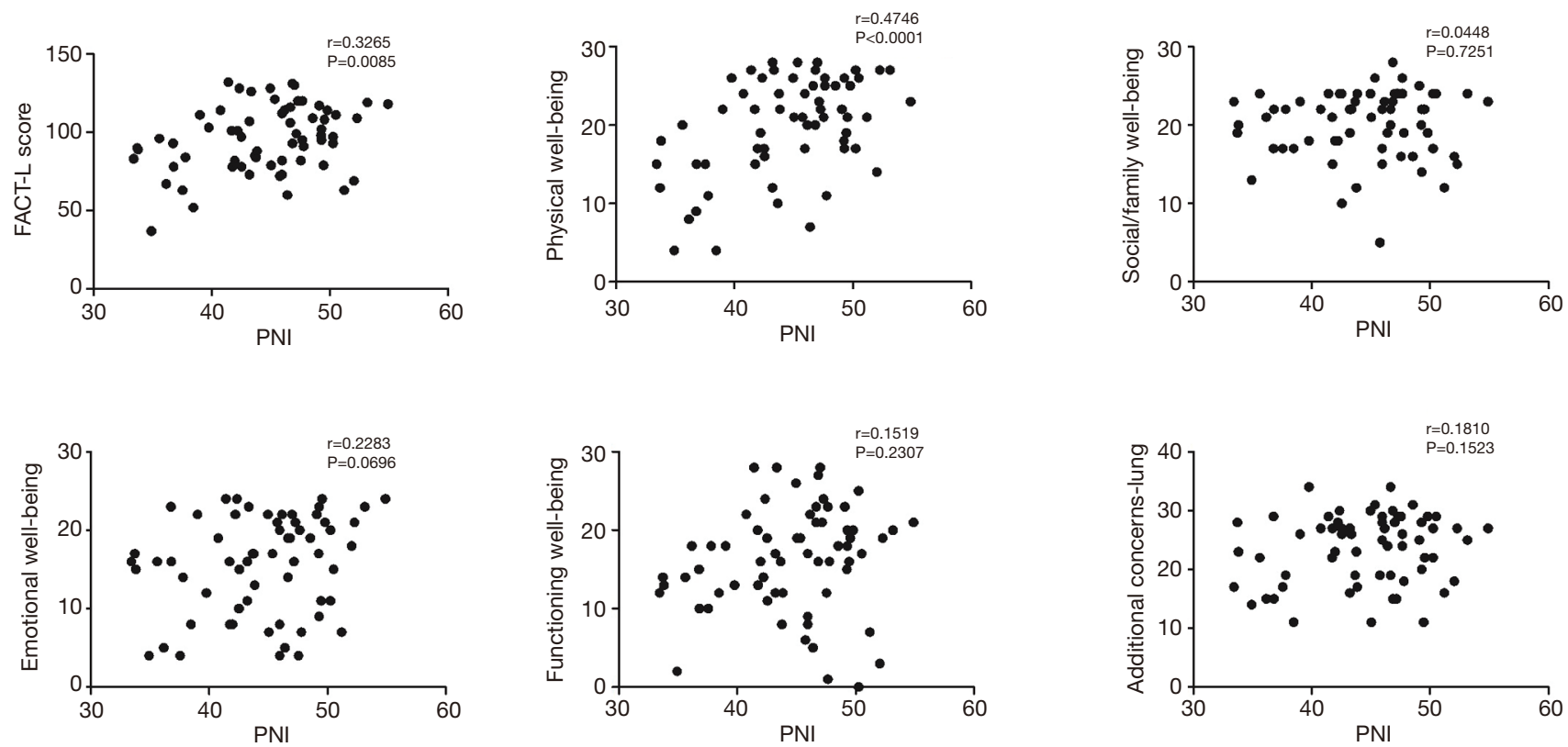

Figure 4 Correlation of PNI and FACT-L score including all subscales in stage IV lung cancer patients. PNI, prognostic nutritional index.

as physical, social, functional, and emotional wellbeing $(21,22)$. Studies have shown that higher baseline/ pretreatment QOL is associated with longer overall survival $(23,24)$, so to some extent improving QOL by effective palliation of cancer-related symptoms is as important as curing the cancer (25).

It has been proposed that QOL should be a routine evaluation in lung cancer care and many instruments have been developed for this purpose (26). However, in daily clinical practice the evaluation is not always performed for every patient because administering and completing questionnaires can be time consuming, which suggests the need for a simple means of identifying patients at high risk of low QOL.

In our study, we found the stage IV lung cancer patients had lower FACT-L scores than the stage III patients, especially for the subscales of PWB, SFWB, EWB, and FWB, which can be explained by distant metastasis of the lung cancer. The most common metastatic sites of lung cancer include the brain, bone and pleura (19), and the fatigue and pain from the cancer lesions, as well as psychological symptoms and comorbidities lead to a decrease in QOL (27).

Malnutrition is common in lung cancer patients because of the tumor itself and the systemic therapy $(28,29)$. It has been reported that approximately $34-69 \%$ of lung cancer patients are malnourished, and the percentage of patients at risk of malnutrition might be even higher (30). Cancerrelated symptoms such as loss of appetite, pain, coughing and fatigue can lead to insufficient energy intake, while abnormal protein and energy metabolism by the cancer cells and aberrant systemic inflammatory reactions result in energy overconsumption.

The PNI was first introduced by Onodera et al. in 1984 to evaluate the risk of postoperative complications according to baseline nutritional status in gastric cancer patients (31). The PNI reflects the nutritional and immune status of patients and has proved to be a reliable predictor of survival in several malignancies including lung cancer (32).

In our study, the average PNI of the late-stage lung cancer patients was $44.24 \pm 5.31$, reflecting impaired nutritional and immune status. The PNI correlated with QOL in stage IV lung cancer patients, especially in the subscale of $\mathrm{PWB}$, which suggests that improving both nutritional and immune status might be beneficial for improving QOL, but further research is needed to confirm this. A possible reason for the correlation between low PNI and poor QOL in stage IV lung cancer patients is that they more frequently develop the cachexia syndrome due to disease severity, which contributes to the state 
of malnutrition through increased fatigue and pain, psychological symptoms and comorbidities, eventually leading to decreased QOL $(33,34)$. Another important factor is immune status: a lower PNI correlates with impaired immune status, which leads to treatment failure and increased comorbidities (35).

However, no statistically significant correlation between the PNI and QOL was observed in stage III lung cancer patients, and a possible explanation would be the difference in disease severity. Greater severity of disease symptoms correlates with lower QOL (36). Stage III lung cancer patients may have less severe symptoms, so the PNI was less able to predict QOL in this group of patients.

There are several limitations in our study. First, this was an observational study with a relatively small number of patients. Second, we focused on the baseline PNI and QOL of late-stage lung cancer patients. A large, prospective study is needed to confirm the relationship of nutrition and immune interventions and QOL.

\section{Conclusions}

PNI was a marker of QOL in stage IV lung cancer patients. Lower PNI may indicate the need for QOL interventions, making it a simple biomarker for use in lung cancer management. However, further study is needed to clarify the exact role of PNI in lung cancer.

\section{Acknowledgments}

Funding: None.

\section{Footnote}

Reporting Checklist: The authors have completed the MDAR reporting checklist. Available at http://dx.doi.org/10.21037/ apm-20-1892

Data Sharing Statement: Available at http://dx.doi. org/10.21037/apm-20-1892

Conflicts of Interest: All authors have completed the ICMJE uniform disclosure form (available at http://dx.doi. org/10.21037/apm-20-1892). The authors have no conflicts of interest to declare.

Ethical Statement: The authors are accountable for all aspects of the work in ensuring that questions related to the accuracy or integrity of any part of the work are appropriately investigated and resolved. All subjects gave written consent after they were fully informed of the study's objectives and methods. The study was approved by the Institutional Research Ethics Committee of the First Affiliated Hospital of Sun Yat-sen University. All subjects were required to be able to independently complete a questionnaire. The study was conducted in accordance with the Declaration of Helsinki (as revised in 2013).

Open Access Statement: This is an Open Access article distributed in accordance with the Creative Commons Attribution-NonCommercial-NoDerivs 4.0 International License (CC BY-NC-ND 4.0), which permits the noncommercial replication and distribution of the article with the strict proviso that no changes or edits are made and the original work is properly cited (including links to both the formal publication through the relevant DOI and the license). See: https://creativecommons.org/licenses/by-nc-nd/4.0/.

\section{References}

1. Siegel RL, Miller KD, Jemal A. Cancer statistics, 2019. CA Cancer J Clin 2019;69:7-34.

2. Ferlay J, Colombet M, Soerjomataram I, et al. Estimating the global cancer incidence and mortality in 2018: GLOBOCAN sources and methods. Int J Cancer 2019;144:1941-53.

3. Bray F, Ferlay J, Soerjomataram I, et al. Global cancer statistics 2018: GLOBOCAN estimates of incidence and mortality worldwide for 36 cancers in 185 countries. CA Cancer J Clin 2018;68:394-424.

4. Xia L, Huang $\mathrm{H}$, Xiao H, et al. Utilization of combined PD-L1 expression and neutrophil-to-lymphocyte ratio prior to surgery as a prognostic factor in non-small cell lung cancer with brain metastasis. Transl Cancer Res 2019;8:2864-77.

5. Ambroggi M, Biasini C, Toscani I, et al. Can early palliative care with anticancer treatment improve overall survival and patient-related outcomes in advanced lung cancer patients? A review of the literature. Support Care Cancer 2018;26:2945-53.

6. Schwartz RM, Alpert N, Rosenzweig K, et al. Changes in quality of life after surgery or radiotherapy in early-stage lung cancer. J Thorac Dis 2019;11:154-61.

7. Pinheiro LC, Reeve BB. Investigating the prognostic ability of health-related quality of life on survival: a prospective cohort study of adults with lung cancer. 
Support Care Cancer 2018;26:3925-32.

8. Maione P, Perrone F, Gallo C, et al. Pretreatment quality of life and functional status assessment significantly predict survival of elderly patients with advanced non-small-cell lung cancer receiving chemotherapy: a prognostic analysis of the multicenter Italian lung cancer in the elderly study. J Clin Oncol 2005;23:6865-72.

9. Harrison JD, Young JM, Price MA, et al. What are the unmet supportive care needs of people with cancer? A systematic review. Support Care Cancer 2009;17:1117-28.

10. Glare PA. Early implementation of palliative care can improve patient outcomes. J Natl Compr Canc Netw 2013;11 Suppl 1:S3-S9.

11. Eton DT, Fairclough DL, Cella D, et al. Early change in patient-reported health during lung cancer chemotherapy predicts clinical outcomes beyond those predicted by baseline report: results from Eastern Cooperative Oncology Group Study 5592. J Clin Oncol 2003;21:1536-43.

12. Efficace F, Bottomley A, Smit EF, et al. Is a patient's selfreported health-related quality of life a prognostic factor for survival in non-small-cell lung cancer patients? A multivariate analysis of prognostic factors of EORTC study 08975. Ann Oncol 2006;17:1698-704.

13. Sánchez-Lara K, Turcott JG, Juarez E, et al. Association of nutrition parameters including bioelectrical impedance and systemic inflammatory response with quality of life and prognosis in patients with advanced non-small-cell lung cancer: a prospective study. Nutr Cancer 2012;64:526-34.

14. Barthelemy N, Streel S, Donneau AF, et al. Screening for malnutrition in lung cancer patients undergoing radiotherapy. Support Care Cancer 2014;22:1531-6.

15. Simons A, Shaffer LG, Hastings RJ. Cytogenetic Nomenclature: Changes in the ISCN 2013 Compared to the 2009 Edition. Cytogenet Genome Res 2013;141:1-6.

16. Zhang YH, Xie FY, Chen YW, et al. Evaluating the Nutritional Status of Oncology Patients and Its Association with Quality of Life. Biomed Environ Sci 2018;31:637-44.

17. Shen Y, Li H, Yuan ZQ, et al. Low pretreatment PNI correlates with worse survival in patients with stage III/ IV NSCLC who received chemotherapy. Neoplasma 2020;67:394-401.

18. Hu Y, Shen J, Liu R, et al. Prognostic value of pretreatment prognostic nutritional index in non-small cell lung cancer: A systematic review and meta-analysis. Int J Biol Markers 2018;33:372-8.

19. Brierley J, Gospodarowicz MK, Wittekind C. TNM classification of malignant tumours. Eighth edition. ed. 2017.

20. Wan C, Zhang C, Cai L, et al. Psychometric properties of the Chinese version of the FACT-L for measuring quality of life in patients with lung cancer. Lung Cancer 2007;56:415-21.

21. Hennessy CH, Moriarty DG, Zack MM, et al. Measuring health-related quality of life for public health surveillance. Public Health Rep 1994;109:665-72.

22. Donovan K, Sanson-Fisher RW, Redman S. Measuring quality of life in cancer patients. J Clin Oncol 1989;7:959-68.

23. Sloan JA, Zhao X, Novotny PJ, et al. Relationship between deficits in overall quality of life and non-small-cell lung cancer survival. J Clin Oncol 2012;30:1498-504.

24. Cooley ME. Symptoms in adults with lung cancer. A systematic research review. J Pain Symptom Manage 2000;19:137-53.

25. Aggarwal A, Lewison G, Idir S, et al. The State of Lung Cancer Research: A Global Analysis. J Thorac Oncol 2016;11:1040-50.

26. Gralla RJ. Quality-of-life evaluation in cancer: The past and the future. Cancer 2015;121:4276-78.

27. Carlsen K, Jensen AB, Jacobsen E, et al. Psychosocial aspects of lung cancer. Lung Cancer 2005;47:293-300.

28. Vaughan VC, Martin P, Lewandowski PA. Cancer cachexia: impact, mechanisms and emerging treatments. J Cachexia Sarcopenia Muscle 2013;4:95-109.

29. van der Meij BS, Schoonbeek CP, Smit EF, et al. Precachexia and cachexia at diagnosis of stage III non-smallcell lung carcinoma: an exploratory study comparing two consensus-based frameworks. Br J Nutr 2013;109:2231-9.

30. Kiss N. Nutrition support and dietary interventions for patients with lung cancer: current insights. Lung Cancer (Auckl) 2016;7:1-9.

31. Onodera T, Goseki N, Kosaki G. Prognostic nutritional index in gastrointestinal surgery of malnourished cancer patients. Nihon Geka Gakkai Zasshi 1984;85:1001-5.

32. Hong $\mathrm{S}$, Zhou T, Fang $\mathrm{W}$, et al. The prognostic nutritional index (PNI) predicts overall survival of small-cell lung cancer patients. Tumour Biol 2015;36:3389-97.

33. Padilla GV. Psychological aspects of nutrition and cancer. Surg Clin North Am 1986;66:1121-35.

34. Tisdale MJ. Mechanisms of cancer cachexia. Physiol Rev 2009;89:381-410.

35. Shoji F, Takeoka H, Kozuma Y, et al. Pretreatment prognostic nutritional index as a novel biomarker in 
non-small cell lung cancer patients treated with immune checkpoint inhibitors. Lung Cancer 2019;136:45-51.

36. Polański J, Jankowska-Polanska B, Uchmanowicz I, et al. Malnutrition and quality of life in patients with non-small-

Cite this article as: Zhang Z, Chen A, Xie F, Li X, Hu G, Lin G. Low prognostic nutrition index predicts poorer quality of life in late-stage lung cancer. Ann Palliat Med 2020;9(6):3976-3984. doi: 10.21037/apm-20-1892 cell lung cancer. Adv Exp Med Biol 2017;1021:15-26.

(English Language Editor: K. Brown) 